\title{
SIRT1 deacetylates mitochondrial trifunctional enzyme a subunit to inhibit ubiquitylation and decrease insulin resistance
}

\author{
Yan-Mei Wang ${ }^{1}$, Ting-Lei Huang ${ }^{2}$, Chao Meng ${ }^{1}$, Jia Zhang ${ }^{1}$ and Ning-Yuan Fang ${ }^{1}$
}

\begin{abstract}
Dysregulation of free acid metabolism is a major contributor to the development of insulin resistance and diabetes. Mitochondrial trifunctional enzyme subunit (MTPa) has a critical role in fatty acid $\beta$-oxidation. However, the association between MTPa and insulin resistance is not definitively known. Here, we aimed to determine how MTPa affects insulin resistance. We tested how MTPa affected glucose uptake in insulin-resistant 3T3-L1 adipocytes and white adipose tissue (WAT) of $\mathrm{db} / \mathrm{db}$ diabetic mice. We also measured how acetylation and ubiquitylation modifications regulated MTPa activation and stability, using quantitative real-time polymerase chain reactions, immunoblotting, and immunoprecipitation. We found that MTPa overexpression promoted glucose uptake via Glut4 translocation to the plasma membrane in 3T3-L1 adipocytes. Moreover, MTPa upregulation decreased glycemia in db/db mice. Deacetylation increased MTPa protein stability and its ability to reduce insulin resistance. The activation of SIRT1, a major deacetylase, prevented MTPa degradation by decreasing its acetylation in adipocytes. Our study demonstrates a new role for MTPa in reducing insulin resistance. Acetylation and ubiquitylation modifications of MTPa were crucial to regulating its function in glucose metabolism.
\end{abstract}

\section{Introduction}

Insulin resistance occurs when a given concentration of insulin produces less than the expected effect on target cells, which can lead to impaired glucose intolerance ${ }^{1}$. Insulin resistance can occur with obesity, pregnancy, burn trauma, and metabolic syndrome, and can cause type 2 diabetes mellitus (T2DM) and cardiovascular dysfunction. Although many researchers study the pathogenesis of insulin resistance ${ }^{2}$, little is known about the underlying mechanism that initiates and advances insulin resistance.

\footnotetext{
Correspondence: Jia Zhang (zhangjiadoctor@126.com) or Ning-

Yuan Fang (fnyuan123@163.com)

'Department of Geriatrics, Ren Ji Hospital, School of Medicine, Shanghai Jiao Tong University, No. 160 Pujian Road, Pudong New Area, Shanghai 200127,

China

${ }^{2}$ Department of Oncology, Ren Ji Hospital, School of Medicine, Shanghai Jiao Tong University, No. 160 Pujian Road, Pudong New Area, Shanghai 200127, China

Edited by A. Peschiaroli
}

Dysregulation of free acid metabolism is a major contributor to insulin resistance and $\mathrm{T}_{2} \mathrm{DM}^{3,4}$. An increase in free fatty acids is associated with defective fatty acid oxidation, which induces or aggravates insulin resistance in adipose, liver, and muscle tissue by directly or indirectly generating metabolites and altering insulin signaling. Free fatty acid reduction is a target for treating insulin resistance ${ }^{5}$.

Mitochondrial trifunctional protein $\alpha$-subunit (MTP $\alpha$ ) is involved in fatty acid $\beta$-oxidation (FAO). MTP $\alpha$ has longchain hydratase activity, which catalyzes the second step of fatty acid $\beta$-oxidation, as well as 3-hydroxyacy-CoA dehydrogenase activity, which catalyzes the third step. A MTP $\alpha$ gene defect causes defective mitochondrial fatty acid oxidation and reverses insulin-stimulated suppression of hepatic glucose production ${ }^{6}$. Furthermore, heterozygous mice lacking MTP $\alpha$ have significantly reduced fatty acid oxidation in liver tissue and develop hepatic steatosis and insulin resistance ${ }^{7}$. Thus, MTP $\alpha$ is a critical enzyme in fatty acid $\beta$-oxidation and may play an important role in insulin 
signaling. However, there is no definitive association between MTP $\alpha$ and insulin resistance.

During diabetes or insulin resistance, excess free fatty acids and high glucose concentrations increase the concentration of TCA cycle products, such as acetyl-CoA and $\mathrm{NADH}^{8,9}$. Increased acetyl-CoA and NADH concentrations induce acetylation of mitochondrial proteins, including $\mathrm{MTP}^{10,11}$. Lysine acetylation is a common protein post-translational modification that regulates cellular metabolism ${ }^{12,13}$. MTP $\alpha$ can be acetylated on three lysine residues 350/383/406, which affects its function in hepatic steatosis ${ }^{14}$. However, the relationship between MTP $\alpha$ acetylation and insulin resistance is unclear.

SIRT1 is an $\mathrm{NAD}^{+}$-dependent deacetylation enzyme. SIRT1 regulates glucose metabolism through its deacetylase activity $^{15}$ and directly or indirectly contributes to insulin signaling ${ }^{16}$. However, elevated glucose levels in T2DM downregulate the protein expression of SIRT1, leading to increased MTP $\alpha$ acetylation. Further, the direct role of SIRT1 in regulating MTP $\alpha$ acetylation remains unknown.

In this study, we demonstrated a novel function for MTP $\alpha$ in reducing insulin resistance. MTP $\alpha$ overexpression promoted insulin-dependent glucose uptake and activated the insulin signaling pathway. We identified MTPo acetylation at four lysine sites (K359, K383, K620, and K625). Mutation of the K625 acetylation site blocked MTP $\alpha$ ubiquitylation and its subsequent degradation. SIRT1 deacetylated MTP $\alpha$ at the K625 site and repressed its degradation. MTP $\alpha$ deacetylation mediated by 9PAHSA or resveratrol, a SIRT1 agonist, decreased insulin resistance.

\section{Materials and methods}

\section{Cell culture and cell differentiation}

3T3-L1 preadipocytes were obtained from the Chinese Academy of Medical Sciences and Peking Union Medical College (Beijing, China). 3T3-L1 preadipocytes were grown in DMEM supplemented with 10\% FBS, $200 \mathrm{U} / \mathrm{ml}$ penicillin and $200 \mathrm{U} / \mathrm{ml}$ streptomycin in $5 \% \mathrm{CO}_{2}$ humidified atmosphere at $37^{\circ} \mathrm{C}$ until confluence. Two days after confluence, to induce adipocyte differentiation, cells were incubated for $48 \mathrm{~h}$ in DMEM supplemented with 10\% FBS containing $500 \mu \mathrm{M}$ 3-isobutyl-1-methylxanthine (IBMX), $0.25 \mu \mathrm{M}$ dexamethasone, and $5 \mu \mathrm{g} / \mathrm{ml}$ insulin. Then the cells were maintained in culture medium supplemented with insulin only, which were changed every 2 days until establishment of insulin resistance.

The SIRT1 activator resveratrol was added to the mature adipocytes during the period of induction of insulin resistance at the final concentration of $10 \mu \mathrm{M}$, while the SIRT1-specific inhibitor EX527 was added at the final concentration of $10 \mu \mathrm{M}$. 9-PAHSA was added at the final concentration of $20 \mu \mathrm{M}$. The proteasomal inhibitor MG132 was added to the mature adipocytes $4 \mathrm{~h}$ before harvest at the final concentration of $10 \mu \mathrm{M}$. For protein stability assay, cells were treated with $100 \mu \mathrm{g} / \mathrm{ml}$ Cycloheximide (CHX, from Sigma) for the indicated time before harvest.

\section{Induction of insulin resistance ${ }^{2}$}

Treatment with recombinant mouse TNF- $\alpha(4 \mathrm{ng} / \mathrm{ml})$ was initiated with mature adipocytes from day 8 of differentiation. Media was changed daily for TNF- $\alpha$ treatment for a total incubation time of 4 days.

\section{Animal studies}

All animal experiments were approved by Fudan University Animal Care and Use Committee and also meet the guidelines of the National Institutes of Health Guide for the Care and Use of Laboratory Animals (UJS-LAER2017042301). Eight to 12-week-old Male C57BL6/J mice, $\mathrm{db} / \mathrm{db}$ mice and their control littermates were purchased from the Model Animal Research Center of Nanjing University (Nanjing, China). At 8 weeks of age, the C57BL/6 mice were given high fat diet (HFD, Shanghai SLAC Company) for 2 months, with normal chow diet mice as control. Mice with random blood glucose $>11.1 \mathrm{mmol} / \mathrm{L}$ were considered as insulin-resistant mice. All mice were housed in colony cages with ad libitum access to food and water. Mice were kept on a $12 / 12 \mathrm{~h}$ light/dark cycle in a temperature-controlled environment.

$\mathrm{Db} / \mathrm{db}$ mice were divided randomly into two groups: control $\mathrm{db} / \mathrm{db}$ group and $\mathrm{db} / \mathrm{db}$ plus 9-PAHSA group $(50 \mathrm{mg} / \mathrm{kg}$ per day). 9-PAHSA $(0.2 \mathrm{ml})$ was given by gavage once per day for 10 days. The control mice were given the same volume of vehicle (50\% PEG 400, 0.5\% Tween 80, 49.5\% H2O).

Each test group included five mice. All mice were euthanized by intraperitoneal injection of $60 \mathrm{mg} / \mathrm{kg}$ sodium pentobarbital. Then abdominal adipose tissue was removed for further study.

\section{Measurement of random glycemia in $\mathrm{db} / \mathrm{db}$ mice}

Blood was collected from the tail vein of each mouse using heparin-coated capillary tubes. Glucose levels were determined using Accu-Check active bands (Roche Diagnostics).

\section{Gene expression analysis}

RNA isolation, reverse transcription, and PCR were performed as described previously ${ }^{17}$. Briefly, trizol reagent was utilized to extract total RNA from 3T3-L1 adipocytes. Then, $1 \mu \mathrm{g}$ of total RNA was subjected to reverse transcription using the PrimeScript ${ }^{\mathrm{TM}}$ RT Reagent kit. Gene expression was evaluated by Quantitative real-time PCR (RT-PCR) analysis using SYBR Green reagents (SYBR® Premix Ex $\left.\mathrm{Taq}^{\mathrm{TM}}\right)$ and the LightCycler ${ }^{\circledR} 480$ Real-Time PCR System (Roche Diagnostics, Basel, Switzerland). 
RT-PCR reactionshad a final volume of $10 \mu \mathrm{l}$. The following cycler program was used for reactions: initial denaturing at $95^{\circ} \mathrm{C}$ for $5 \mathrm{~min}$, followed by 45 cycles of denaturing at $95^{\circ} \mathrm{C}$ for $10 \mathrm{~s}$ and annealing and extension at $60^{\circ} \mathrm{C}$ for $20 \mathrm{~s}$. The threshold cycle $(\mathrm{Ct})$ value was computed for each amplification curve, and $\Delta \mathrm{Ct}$ values were calculated by subtracting the $\mathrm{Ct}$ value for $\beta$-actin RNA from the $\mathrm{Ct}$ value for each experimental sample. The results were expressed as fold-changes with respect to the control using the 2- $\Delta \Delta \mathrm{CT}$ formula. The primer sequences for qPCR were as follows: MTP $\alpha, 5^{\prime}$-ACA TCG GAG CTG TCT TTG GG-3' (forward) and 5'-GAC TCG TAC TTC CGT AGC CG-3' (reverse); $\beta$-actin, $5^{\prime}$-AGC CTT GTA GGT ACC CAA CC-3' (forward) and 5'-TCC CAC TCA CCT GAG GTG CTG AA-3' (reverse).

\section{Glucose uptake assay}

Glucose uptake assay in 3T3-L1 adipocytes were performed as described previously ${ }^{18}$. Briefly, cells in 96 well dishes were washed twice with PBS and incubated with $100 \mu \mathrm{KRPH} / 2 \%$ BSA for $40 \mathrm{~min}$. Prepare sample background controls, insulin stimulated cells and nonstimulated control samples. (1) Sample background control (untreated) cells: Do not add insulin and 2deoxyglucose (2-DG). (2) Insulin stimulated cells: $\mathrm{KRPH} / 2 \% \mathrm{BSA}$ contained with $10 \mu \mathrm{M}$ insulin for $20 \mathrm{~min}$ and add $10 \mu \mathrm{l}$ of $10 \mathrm{mM}$ 2-DG for $20 \mathrm{~min}$. (3) Nonstimulated control samples: Non-insulin stimulated cells, but add $10 \mu \mathrm{l}$ of $10 \mathrm{mM}$ 2-DG for $20 \mathrm{~min}$. Prepare Reaction Mix A and add in all samples. And incubate for 1 hour. Add $90 \mu$ l Extraction buffer in each well and heat at $90^{\circ} \mathrm{C}$ for $40 \mathrm{~min}$. Prepare Reaction Mix B fresh and add $38 \mu \mathrm{l}$ in all wells. Measure output OD at $412 \mathrm{~nm}$ wavelength on a microplate reader in a kinetic mode, every 2-3 min, at $37^{\circ} \mathrm{C}$ protected from light.

\section{Immunofluorescence staining}

Immunofluorescence staining for the Glut4 membrane translocation analysis was conducted as described previously $^{18}$. Briefly, cells were blocked with 5\% BSA for $30 \mathrm{~min}$ at room temperature with membrane rupture treatment by Triton to detect total Glut4 or without membrane rupture to determine membrane distribution. Cells were incubated at $4{ }^{\circ} \mathrm{C}$ with anti-Glut 4 antibody overnight. Equal PBS was added instead of Glut4 as a negative control. The Cy3-conjugated secondary antibody was applied to the samples at room temperature for $1 \mathrm{~h}$. After washing with PBS, images were immediately captured under an immunofluorescence microscope.

To investigate the subcellular localization of SIRT1 in 3T3-L1 adipocytes, adipocytes were stained with MitoTracker (Molecular Probes), together with specific antibody for SIRT1 detection. Images were captured under an immunofluorescence microscope.

\section{Immunoprecipitation (IP)}

IP was performed in lysates prepared from 3T3-L1 adipocytes $(100 \mu \mathrm{g}$ total protein) using either the acetyLys antibody, Ub antibody or normal rabbit IgG at $4{ }^{\circ} \mathrm{C}$ overnight. On the next morning, the protein-antibody complex was incubated with $15 \mu \mathrm{l}$ magnetic protein A + $\mathrm{G}$ beads for $1 \mathrm{~h}$ at $4{ }^{\circ} \mathrm{C}$ with gentle rotation. The antibodyprotein-bead complexes then were washed three times with IP buffer. The protein in the complex then was eluted with $30 \mu \mathrm{l} 1 \times$ loading buffer and boiled before running on a $12 \%$ SDS-polyacrylamide gel. The proteins were transferred to nitrocellulose membranes, and acetyLys/Ub-associated MTP $\alpha$ proteins were immunoblotted using antibodies against MTP $\alpha$.

\section{RNA interference}

For MTP knockdown induction in vitro, the 3T3-L1 preadipocytes were transfected with control shRNA (shCON) or MTP $\alpha$-targeted shRNA (shMTP $\alpha$ ) lentiviral particles. The target sequence used against mouse MTP $\alpha$ was as follows: 5'-TCTCCCAATCAATCAAATT-3'; and the sequence of the control shRNA was as follows: $5^{\prime}$ TTCTCCGAACGTGTCACGT-3'. For MTP $\alpha$ overexpression induction in vitro, 3T3-L1 preadipocytes were transduced with lentiviral vector containing an MTP $\alpha$ (ovMTP $\alpha$ ) expression cassette. Control cells were transfected with the control vector (ovCON). For SIRT1 knockdown induction in vitro, the 3T3-L1 preadipocytes were transfected with control shRNA (shCON) or SIRT1targeted shRNA (shSIRT1) lentiviral particles. The target sequence used against mouse SIRT1 was as follows: $5^{\prime}-$ CCCTCAAGCCATGTTTGAT- $3^{\prime}$; and the sequence of the control shRNA was as follows: $5^{\prime}$-TTCTCCGAA CGTGTCACGT-3'. For the mutation of MTP acetylation sites induction in vitro, 3T3-L1 preadipocytes were transduced with lentiviral vector containing each lysine mutation to an arginine (R) (K359R, K383R, K620R, K625R). The lentiviral vector and particles were constructed and synthesized by GeneChem (Shanghai, China).

Lentiviral-transfected 3T3-L1 preadipocytes were induced to differentiate into mature adipocytes and then used as cell model throughout the experiments.

\section{SIRT3 siRNA knockdown in adipocytes}

3T3-L1 adipocytes transfected with non-targeting siRNA or SIRT3-targeting siRNA (GeneChem, Shanghai, China) using the Lipo3000 transfection reagent following the manufacturer's instructions (Life Technologies, Carlsbad, CA, USA). The knockdown efficiency of SIRT3targeting siRNA was evaluated by western blotting $72 \mathrm{~h}$ post-transfection. siRNA sequences are as follows: $5^{\prime}$ CAGCUUGUCUGAAGCAGUATT $-3^{\prime}$ and the sequence of the control siRNA was as follows: $5^{\prime}$-UUCUCC GAACGUGUCACGUTT-3'. SIRT3 siRNA-transfected 
3T3-L1 adipocytes were treated with10 $\mu \mathrm{M}$ resveratrol for 4 days.

\section{Mass spectrometry analysis}

The peptide samples were analyzed on Thermo Fisher LTQ Obitrap ETD mass spectrometry, Briefly, loaded sample onto an HPLC chromatography system named Thermo Fisher Easy-nLC 1000 equipped with a C18 colume $(1.8 \mathrm{~mm}, 0.15 \times 1.00 \mathrm{~mm})$. Solvent A contained $0.1 \%$ formic acid and solvent B contained $100 \%$ acetonitrile. The elution gradient was from 4 to $18 \%$ in $182 \mathrm{~min}$, 18 to $90 \%$ in $13 \mathrm{~min}$ solvent $\mathrm{B}$ at a flow rate of $300 \mathrm{nl} / \mathrm{min}$. Mass spectrometry analysis were carried out carried out at the AIMS Scientific Co.,Ltd.(Shanghai, China) in the positive-ion mode with an automated data-dependent MS/MS analysis with full scans $(350-1600 \mathrm{~m} / \mathrm{z})$ acquired using FTMS at a mass resolution of 30,000 and the ten most intense precursor ions were selected for MS/MS. The MS/MS was acquired using higher-energy collision dissociation at $35 \%$ collision energy at a mass resolution of 15,000 .

\section{SIRT1 deacetylase activity assay}

SIRT1 deacetylase activity was assessed using a commercial fluorometric assay kit (cat. no. CS1040; SigmaAldrich). 9-PAHSA (100 nmol, $200 \mathrm{nmol}, 400 \mathrm{nmol}$ ) were incubated with the SIRT1 enzyme, SIRT1 substrate and NAD + to screen the activator of SIRT1. Moreover, an inhibitor (nicotinamide) and an activator (resveratrol) as negative and positive controls, respectively. Protein $(100 \mu \mathrm{g})$ were extracted from 3T3-L1 adipocytes or adipose tissue in $\mathrm{db} / \mathrm{db}$ mice to detect SIRT1 deacetylase activity. The fluorescence emitted, due to deacetylation of the substrate by SIRT1, was measured at $350 \mathrm{~nm}$ excitation and $450 \mathrm{~nm}$ emission wavelengths using a fluorescence microplate reader (SpectraMax ${ }^{\circledR}$ M5; Molecular Devices, LLC, Sunnyvale, CA, USA).

\section{Isolation of mitochondria from 3T3-L1 adipocytes}

Isolation of mitochondria from adipocytes was performed using Mitochondria Isolation Kit (Sigma-Aldrich). Enriched fractions of mitochondria from adipocytes were purified also according to the protocol from this kit. The possible contamination of mitochondria with nuclei components was excluded by carrying out western blot analysis of $\mathrm{H} 2 \mathrm{~B}$, a much abundant nuclear protein. The effective mitochondria isolation was assessed by anti-VDAC1.

\section{Protein preparation and western blotting analysis}

Total protein was extracted with RIPA buffer containing phenylmethylsulfonyl fluoride (PMSF) and Halt Protease and Phosphatase Inhibitor Cocktail. Membrane protein was extracted by membrane and cytosol protein extraction kit (Beyotime Biotechnology, China). The concentration of proteins was tested using the bicinchoninic acid (BCA) protein assay. Protein samples $(30 \mu \mathrm{g})$ were separated by SDS-PAGE and then transferred to nitrocellulose membranes (Bio-Rad, Richmond, CA, USA). Membranes were blocked in 5\% non-fat milk in Tris-buffered saline containing $0.05 \%$ Tween-20 (TBST) for $1 \mathrm{~h}$ at room temperature. Then, membranes were incubated with primary antibody at $4{ }^{\circ} \mathrm{C}$ overnight. Anti-MTP $\alpha$, anti-IRS1/P-IRS1, anti-Glut4, anti- $\beta$-actin, anti-GAPDH, anti-SIRT1, antiAce, anti-Ub and anti- $\mathrm{Na}^{+}$-ATPase $\alpha-1$ were from Abcam. Anti-Akt/P-Akt, anti-VDAC1, anti-H2B and secondary antibody were from Cell Signaling Technology. Probed membranes were washed several times with TBST, and then incubated with horseradish peroxidase conjugated secondary antibodies at room temperature for $1 \mathrm{~h}$. Bound antibody was detected with enhanced chemiluminescence (Millipore, Billerica, MA, USA). Protein expression was quantified using Image J software (NIH, USA). Total protein expression was normalized with respect to $\beta$-actin/GAPDH expression and membrane protein was normalized with respect to $\mathrm{Na}^{+}$-ATPase $\alpha-1$ expression.

\section{Statistical analysis}

All experiments were repeated at least three times. All data were analyzed using GraphPad Prism software (GraphPad Software Inc., CA, USA) and expressed as the mean \pm standard error (SE). One-way ANOVA and twoway ANOVA were used to compare differences among multiple groups, and the non-paired $t$-test was used to analyze two groups after homogeneity of variance testing. A value of $p<0.05$ was considered statistically significant.

\section{Results}

\section{Establishment of insulin resistant cell model}

First, we treated 3T3-L1 adipocytes with TNF- $\alpha(4 \mathrm{ng} / \mathrm{ml})$ to create an insulin resistance (IR) model. We assessed the model by measuring how much insulin was needed to stimulate glucose uptake. TNF- $\alpha$ decreased insulin-dependent glucose uptake. However, the decrease was rescued by pioglitazone, a member of the thiazolidinedione (TZD) class of insulin-sensitizing drugs (Fig. 1a).

We also examined how TNF- $\alpha$ affected insulin signaling. IRS1 and Akt activation are major phosphorylationdependent signaling intermediates in insulin sensitivity. TNF- $\alpha$ treatment decreased insulin-stimulated IRS1 phosphorylation at Thr896 and Akt phosphorylation at Ser473; in contrast, co-treatment with pioglitazone largely prevented TNF- $\alpha$ effects on Akt phosphorylation, while did not reverse TNF- $\alpha$-inhibited IRS-1 phosphorylation (Fig. 1b, c). TNF- $\alpha$ alone or together with pioglitazone had no effect on total Glut4 levels. However, the expression levels of Glut 4 on cell surface were decreased in TNF- $\alpha$ treated 3T3-L1 adipocytes compared with control based on western blotting detection. In contrast, pioglitazone 
a

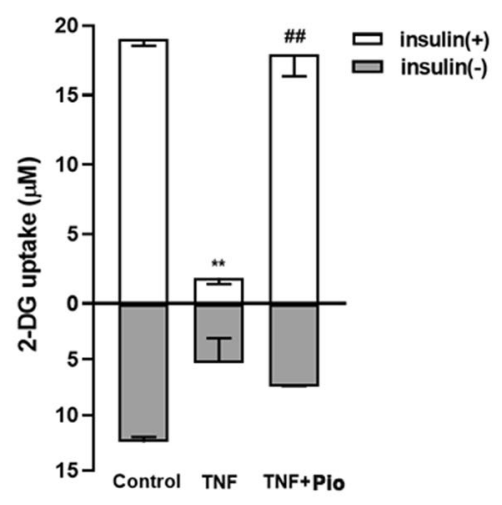

b

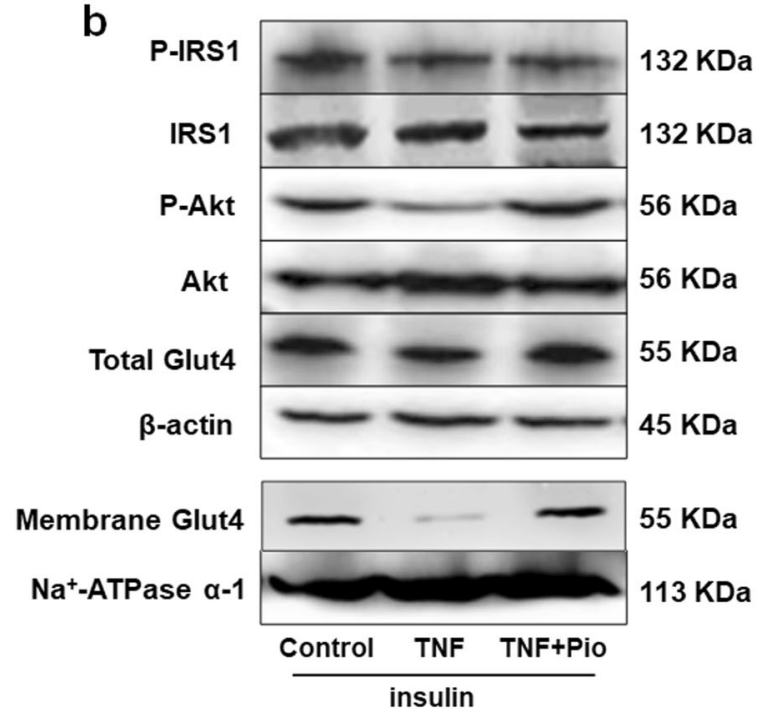

C

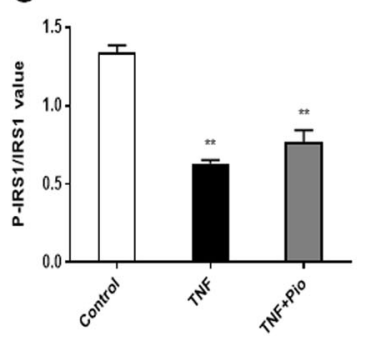

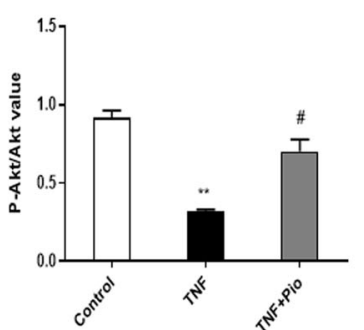
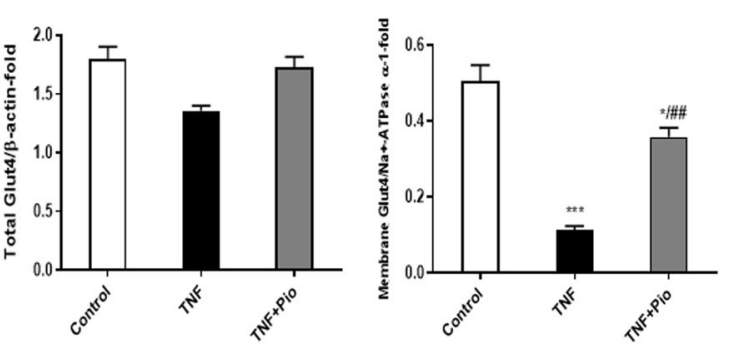

Fig. 1 Establishment of insulin resistant state in 3T3-L1 adipocytes. a Glucose uptake in 3T3-L1 adipocytes. Basal glucose transport (gray) and insulin stimulated glucose uptake (white) are shown. Adipocytes were untreated, treated with TNF-a (4 ng/ml) alone or TNF-a (4 ng/ml) plus pioglitazone $(2 \mu \mathrm{M})$ for 4 days. ${ }^{* *} p<0.01$ vs. insulin alone, ${ }^{*} p<0.05$ vs. TNF-a plus insulin (one-way ANOVA). b Insulin signaling parameters were examined by western blotting analysis in 3T3-L1 adipocytes. Adipocytes were untreated, treated with TNF-a (4 ng/ml) alone or TNF-a (4 ng/ml) plus pioglitazone $(2 \mu \mathrm{M})$ for 4 days. All adipocytes were treated with insulin $(5 \mu \mathrm{g} / \mathrm{ml})$ for 4 days. c Quantification of western blotting. ${ }^{*} p<0.05$, ${ }^{* *} p<0.01$, ${ }^{* * *} p<0.001$ vs. control, ${ }^{\#} p<0.05,{ }^{\# \#} p<0.01$ vs. TNF-a (one-way ANOVA). Data are representative of at least three different experiments. All data represent means \pm standard error (SE).

treatment significantly increased membrane Glut4 levels, suggesting that pioglitazone promoted insulin-stimulated Glut4 plasma membrane translocation (Fig. 1b, c). Altogether, these results indicated that we successfully established the IR model in 3T3-L1 adipocytes.

\section{MTPa increased glucose uptake and activated insulin} signaling pathway in TNF-a-induced insulin-resistant 3T3L1 adipocytes

We then examined MTP $\alpha$ expression in the IR model in 3T3-L1 adipocytes. Protein expression of MTP $\alpha$ was reduced in TNF- $\alpha$-treated 3T3-L1 adipocytes, whereas mRNA expression was not significantly altered (Fig. 2a, b). We next sought to extend these observations to an in vivo IR model, using mice with high fat diet (HFD)-induced IR and $\mathrm{db} / \mathrm{db}$ mice deficient for the leptin receptor. Results showed that MTP $\alpha$ protein levels were significantly lower in white adipose tissue (WAT) of HFD-induced IR mice
(Fig. 2c) and db/db diabetic mice (Fig. 2d) than in control mice. However, mRNA expression was not significantly altered (Fig. 2a).

Next, we evaluated the role of MTP $\alpha$ in IR. We transfected 3T3-L1 preadipocytes with a lentiviral system that induced MTP $\alpha$ overexpression (ovMTP $\alpha$ ). Western blotting showed about 50\% transfection efficiency of ovMTP $\alpha$ in 3T3-L1 preadipocytes (Fig. 2e). OvMTP $\alpha$ attenuated TNF- $\alpha$-induced IR, as evidenced by a dramatical increase in insulin-stimulated glucose uptake in TNF- $\alpha$-treated 3T3-L1 adipocytes ( $p<0.01$, Fig. 2g). Further, ovMTP $\alpha$ in TNF- $\alpha$ treated 3T3-L1 adipocytes completely blocked the effect of TNF- $\alpha$ and enhanced insulin-stimulated IRS1 and Akt phosphorylation (Fig. 2h).

We also transfected 3T3-L1 preadipocytes with a shRNA lentiviral vector targeting MTP $\alpha$ mRNA ( $\operatorname{shMTP} \alpha)$, which stably knocked down MTP $\alpha$ expression in 3T3-L1 preadipocytes. Western blotting confirmed about $80 \%$ 
a

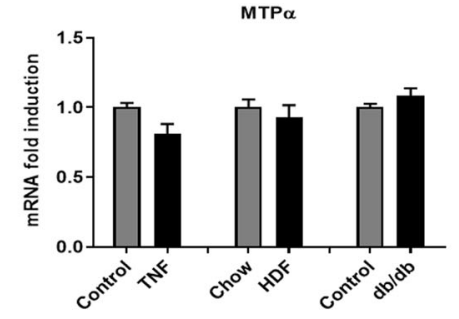

b
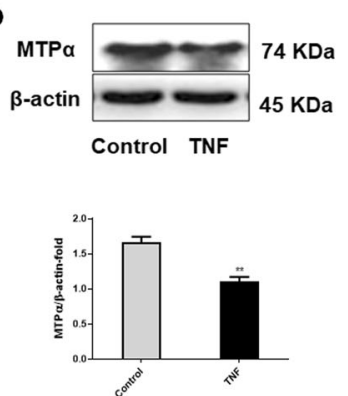

e
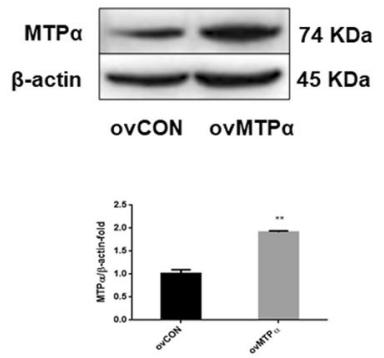

h
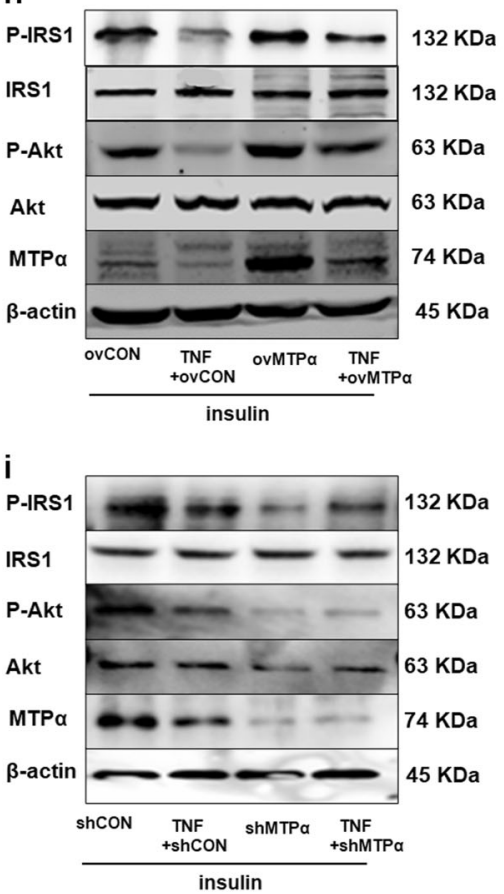

f
C
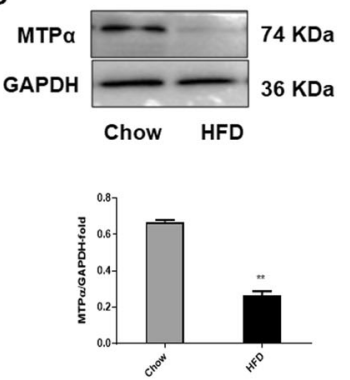
MTPa GAPDH
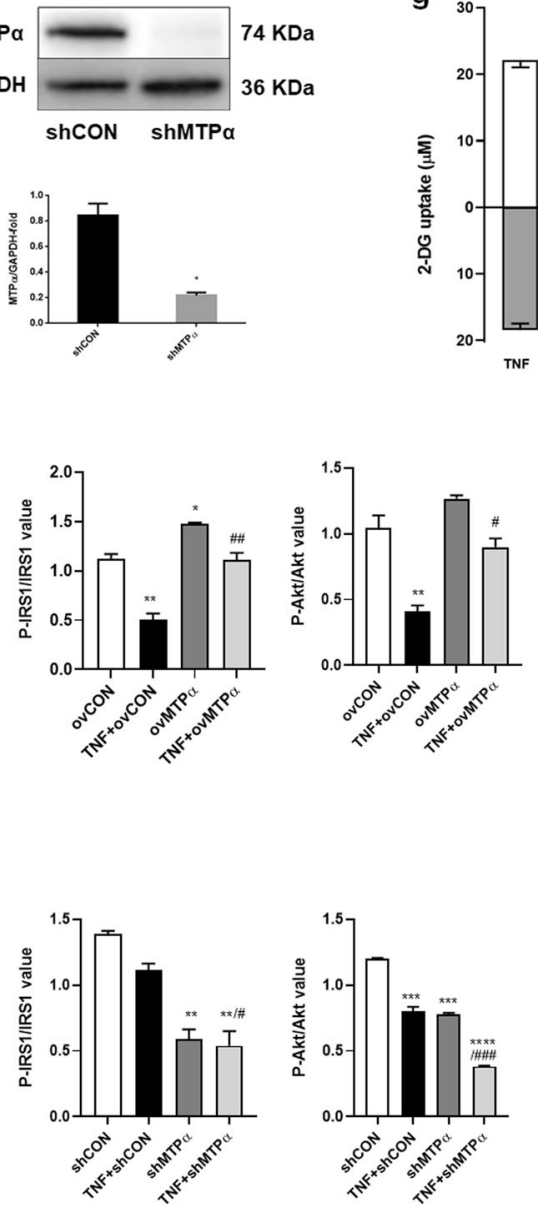
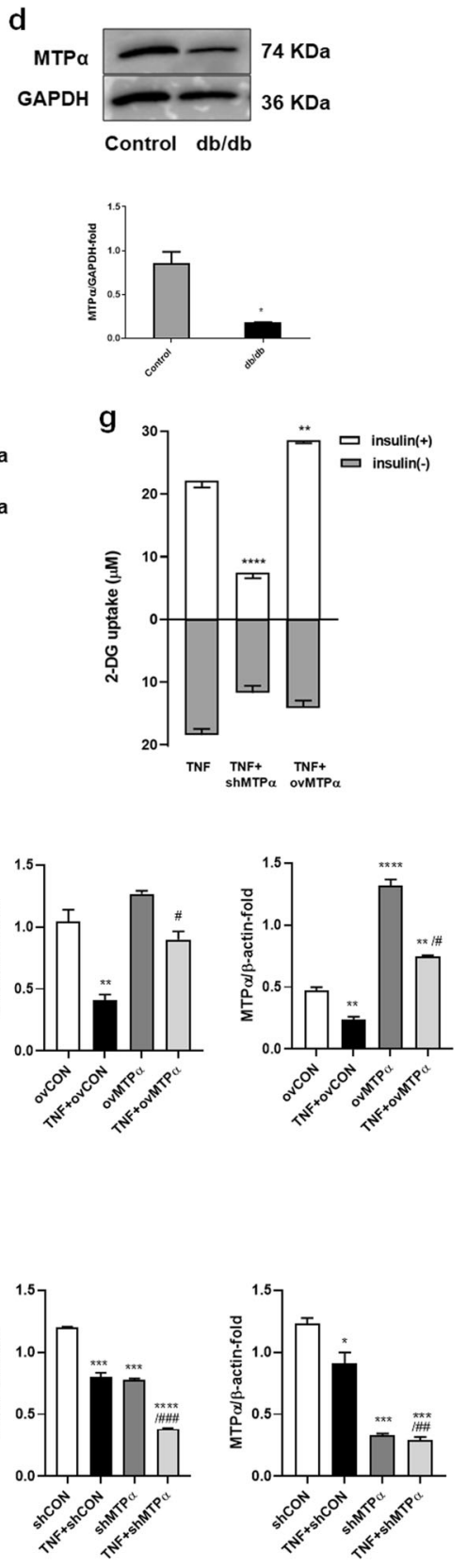

ThMTPa OUMTPa

Fig. 2 (See legend on next page.) 


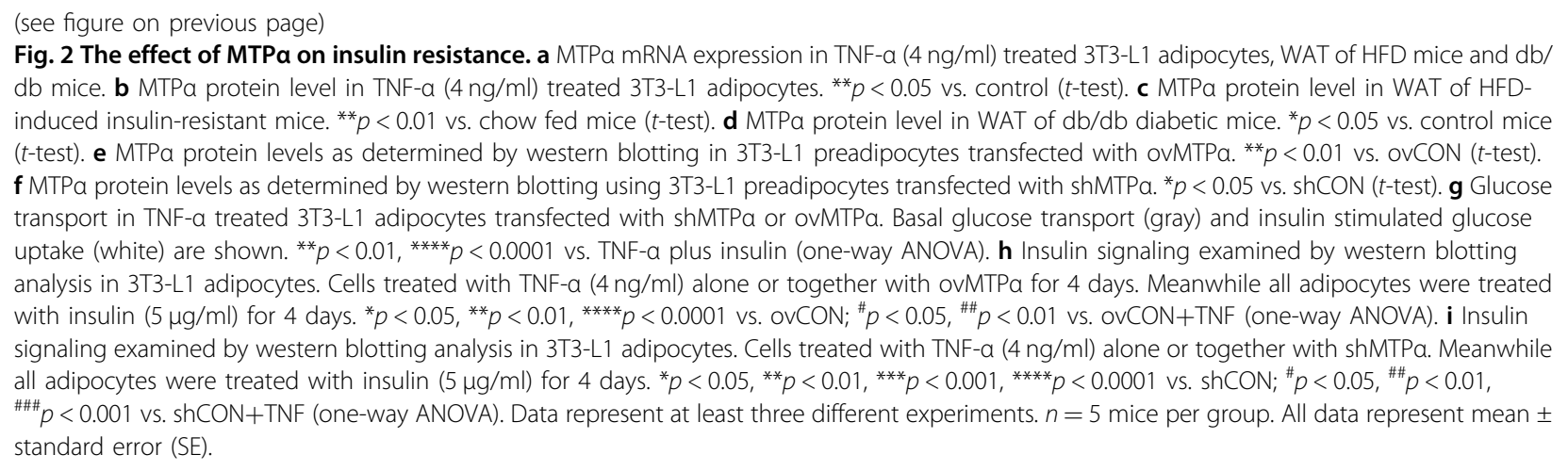

knockdown efficiency of MTPa (Fig. 2f). MTP $\alpha$ knockdown enhanced TNF- $\alpha$-induced insulin resistance, as evidenced by significantly decreased insulin-stimulated glucose uptake in TNF- $\alpha$-treated 3T3-L1 adipocytes $(p<$ 0.0001, Fig. 2g). Moreover, shMTP $\alpha$ treatment further decreased insulin-stimulated phosphorylation of IRS1 and Akt in TNF- $\alpha$ treated 3T3-L1 adipocytes (Fig. 2i). Taken together, these data indicate that MTP $\alpha$ overexpression reduced IR induced by TNF- $\alpha$, whereas MTP $\alpha$ knockdown increased IR.

\section{MTPa promoted insulin-stimulated Glut4 translocation to the plasma membrane in 3T3-L1 adipocytes}

To further determine how glucose uptake was enhanced by MTP $\alpha$, we analyzed MTP $\alpha$ effects on Glut4 translocation to the plasma membrane in 3T3-L1 adipocytes. TNF- $\alpha$ treatment alone or together with shMTP $\alpha$ or ovMTP $\alpha$ had no effect on total Glut4 expression in 3T3L1 adipocytes (Fig. 3a, b). However, TNF- $\alpha$ treatment decreased insulin-stimulated Glut4 protein levels in the plasma membrane of 3T3-L1 adipocytes compared to controls. MTP $\alpha$ overexpression increased insulinstimulated Glut4 protein levels to nearly baseline levels. In contrast, MTP $\alpha$ knockdown further decreased insulinstimulated Glut4 protein levels in the plasma membrane of TNF- $\alpha$-treated 3T3-L1 adipocytes (Fig. 3a-c). These results suggest that MTP $\alpha$ promoted Glut4 translocation.

\section{SIRT1 reversed IR via an MTPa-related pathway}

Resveratrol activates SIRT1 to reverse insulin resistance $^{19}$. To investigate the mechanism underlying SIRT1 anti-diabetic activity, we added the SIRT1 activator resveratrol to TNF- $\alpha$-induced insulin-resistant adipocytes. Resveratrol increased insulin-stimulated glucose uptake in TNF- $\alpha$-induced insulin-resistant 3T3-L1 adipocytes (Fig. 4a). We performed the same experiment in 3T3-L1 adipocytes with MTP $\alpha$-knockdown. The results showed that shMTP $\alpha$ prevented the resveratrol-induced increase in insulin-dependent glucose uptake. We also examined how resveratrol and shMTP $\alpha$ affected insulin signaling, finding that shMTP $\alpha$ decreased the resveratrolinduced insulin stimulated phosphorylation of Akt and IRS1 (Fig. 4b). These results indicate that resveratrol reduced IR via an MTP $\alpha$-related pathway.

To confirm whether SIRT1 reversed IR through MTP $\alpha$, we used 9-PAHSA, a novel activator of SIRT1 that is an endogenous mammalian lipid with anti-diabetic effects ${ }^{20}$. We found that 9-PAHSA significantly increased SIRT1 activity in a dose-dependent manner (Fig. 4c). Further, 9PAHSA treatment in 3T3-L1 adipocytes increased SIRT1 activity, similar to the effect of resveratrol treatment (Supplementary Fig. S1). Also similar to resveratrol, 9PAHSA increased insulin-stimulated glucose uptake in insulin-resistant 3T3-L1 adipocytes. However, this action of 9-PAHSA was reversed by shMTPa (Fig. 4d). Notably, oral administration of 9-PAHSA in $\mathrm{db} / \mathrm{db}$ mice lowered basal glycemia (Fig. 4e). Mechanistically, 9-PAHSA administration increased SIRT1 activation and increased MTP $\alpha$ protein levels in WAT of db/db mice (Fig. 4f, g).

\section{SIRT1 increased MTPa protein levels by inhibiting MTPa ubiquitylation}

To investigate interactions between MTP $\alpha$ and SIRT1, we first examined SIRT1 expression in 3T3-L1 adipocytes

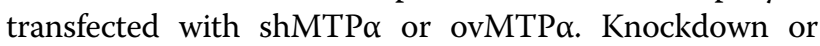
overexpression of MTP $\alpha$ had no effect on SIRT1 protein levels (Fig. 5a). We then examined MTPa expression in 3T3-L1 adipocytes transfected with shSIRT1. The results showed that SIRT1 knockdown decreased MTP $\alpha$ protein expression (Fig. 5b). Treatment of EX527, the SIRT1specific inhibitor, in 3T3-L1 adipocytes also decreased MTP $\alpha$ protein expression, although the change was not significant. Moreover, resveratrol, the SIRT1 activator, increased MTP $\alpha$ protein expression (Fig. 5c). Considering resveratrol also activated the mitochondrial sirtuin, SIRT3, we tested the the role of resveratrol in MTP $\alpha$ protein expression by knocking down SIRT3. The results showed that resveratrol treatment also increased MTP $\alpha$ protein 


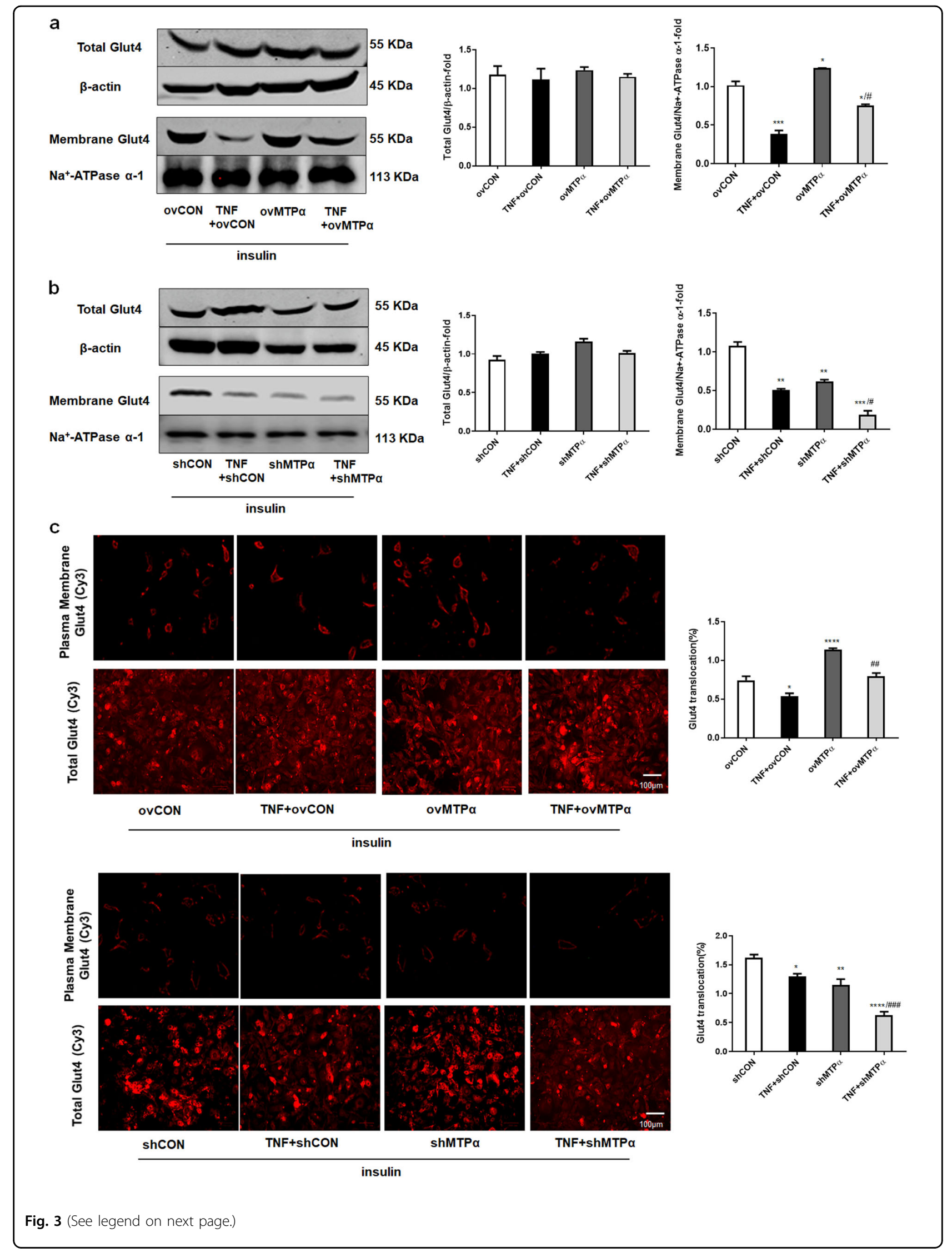


(see figure on previous page)

Fig. 3 MTPa promoted insulin stimulated Glut4 translocation in 3T3-L1 adipocytes. a Total Glut4 protein levels and plasma membrane Glut4 protein levels were measured by western blotting in 3T3-L1 adipocytes transfected with control siRNA or ovMTPa, treated with or without TNF-a $(4 \mathrm{ng} / \mathrm{ml})$ for 4 days. Meanwhile all adipocytes were treated with insulin $(5 \mu \mathrm{g} / \mathrm{ml})$ for 4 days. ${ }^{*} p<0.05,{ }^{* * *} p<0.001$ vs. ovCON; ${ }^{*} p<0.05$ vs. TNF+ ovCON (one-way ANOVA). b Total Glut4 protein levels and plasma membrane Glut4 protein levels were measured by western blotting in 3T3-L1 adipocytes transfected with control siRNA or shMTPa, treated with or without TNF-a $(4 \mathrm{ng} / \mathrm{ml})$ for 4 days. Meanwhile insulin $(5 \mu \mathrm{g} / \mathrm{ml})$ was added into media. ${ }^{* *} p<0.05,{ }^{* * *} p<0.001$ vs. shCON; ${ }^{*} p<0.05$ vs. TNF + shCON (one-way ANOVA). c Immunofluorescence detection of Glut4 plasma membrane translocation in 3T3-L1 adipocytes transfected with control siRNA, shMTPa or ovMTPa and treated with or without TNF-a (4 ng/ml) for 4 days. Meanwhile insulin $(5 \mu \mathrm{g} / \mathrm{ml})$ was added into media. Scale bar: $100 \mu \mathrm{m}$. ${ }^{*} p<0.05,{ }^{* *} p<0.01,{ }^{* * *} p<0.001,{ }^{* * * *} p<0.0001 \mathrm{vs}$. ovCON or shCON; ${ }^{\# \#} p<0.01,{ }^{\# \#} p<0.001$ vs. TNF + ovCON or TNF + shCON (one-way ANOVA). Data represent at least three different experiments. All data represent means \pm standard error (SE).

expression in SIRT3 knockdown cells (Supplementary Fig. S2), further indicating a specific interaction between SIRT1and MTP $\alpha$. Notably, MTP $\alpha$ mRNA levels were not affected by shSIRT1, resveratrol or EX-527 treatment (Fig. 5d, e). It is indicated that SIRT1 may regulate MTP $\alpha$ by altering post-translational modification.

In first, we investigated and observed a physical interaction between SIRT1and MTP $\alpha$. It is acknowledged that MTP $\alpha$ localizes to the mitochondrial. SIRT1 is also found in mitochondria using confocal microscopy (Supplementary Fig. S3a). Protein extracts of purified mitochondria from adipocytes further displayed the presence of SIRT1 in mitochondria (Supplementary Fig. S3b).

We then explored the mechanism underlying SIRT1mediated upregulation of MTP $\alpha$. Our results showed that the proteasomal inhibitor MG132 significantly increased MTP $\alpha$ protein levels $(p<0.05$, Fig. $5 \mathrm{~h})$, indicating that the ubiquitin proteasome pathway degrades MTP $\alpha$. Inhibition of protein synthesis with cycloheximide (CHX) showed that MTP $\alpha$ is an unstable protein with a half life of about $8 \mathrm{~h}$. Activating SIRT1 by resveratrol substantially extended the half life of MTP $\alpha$, while blocking SIRT1 activity by EX-527 or SIRT1 knockdown had adverse effects (Fig. 5f, g). Our data further demonstrate that EX527 treatment increased MTP $\alpha$ ubiquitylation, but resveratrol treatment decreased it (Fig. 5i). Further, SIRT1 knockdown increased MTP $\alpha$ ubiquitylation (Fig. 5i).

To confirm these findings, we also measured MTPa ubiquitylation in 9-PAHSA-treated 3T3-L1 adipocytes and in WAT from 9-PAHSA-treated $\mathrm{db} / \mathrm{db}$ mice. Similarly, 9-PAHSA treatment suppressed MTP $\alpha$ ubiquitylation and increased MTP $\alpha$ protein levels. However, shSIRT1 decreased this effect of 9-PAHSA treatment (Fig. 5j, k). Taken together, these data suggest that SIRT1 repressed MTP $\alpha$ ubiquitylation and subsequent degradation.

\section{SIRT1 mediated MTPa deacetylation, thus inhibiting MTPa ubiquitylation and reducing IR}

SIRT1 has deacetylation enzymatic activity. To identify posttranslational modifications of MTP $\alpha$ regulated by SIRT1, we measured MTP $\alpha$ acetylation after immunoprecipitating MTP $\alpha$ and western blotting with anti-acetyl lysine antibody. We found that EX527 treatment increased MTPa acetylation and resveratrol treatment decreased MTP $\alpha$ acetylation, relative to controls (Fig. 6a). We also detected more MTP $\alpha$ acetylation in shSIRT1 transfected 3T3-L1 adipocytes than in shCON adipocytes (Fig. 6a). Furthermore, 9-PAHSA treatment decreased MTP $\alpha$ acetylation in both 3T3-L1 adipocytes and WAT of $\mathrm{db} / \mathrm{db}$ mice. However, shSIRT1 reversed this decreased MTP $\alpha$ acetylation in 3T3-L1 adipocytes (Fig. 6b, c). These data demonstrate that SIRT1 regulated MTP $\alpha$ acetylation.

To identify MTP $\alpha$ acetylation sites, we conducted mass spectrometry analysis on 3T3-L1 adipocytes. We identified four acetylation sites on MTP $\alpha$ (Fig. 6d). To determine which MTP $\alpha$ acetylation sites were regulated by SIRT1, we mutated each candidate lysine to an arginine (R). We transfected 3T3-L1 adipocytes with wild type, ovMTP $\alpha$ (positive control), or MTP $\alpha$ mutants, treated cells with EX-527, and then performed immunoprecipitation. K625 mutation greatly decreased MTP $\alpha$ acetylation, whereas mutation of K383, K620 or K359 had no effect on MTP $\alpha$ acetylation (Fig. 6e). In addition, MTP $\alpha$ acetylation was higher in ovMTP $\alpha$ transfected adipocytes treated with EX-527 than in untreated cells. However, EX527 treatment did not further increase MTP $\alpha$ acetylation when the K625 site was mutated (Fig. 6f), suggesting that K625 is the MTP $\alpha$ acetylation site regulated by SIRT1.

We also found that SIRT1-mediated MTP $\alpha$ acetylation affected MTPa ubiquitylation. As shown in Fig. 5, inhibiting SIRT1 deacetylases with EX-527 increased MTP $\alpha$ ubiquitylation. Further, activating SIRT1 deacetylases with resveratrol or 9-PAHSA reduced MTP $\alpha$ ubiquitylation. This finding led us to investigate potential crosstalk between MTP $\alpha$ acetylation and ubiquitylation. We found that K625 mutation decreased MTP $\alpha$ ubiquitylation levels. Furthermore, EX-527 increased ovMTP $\alpha$ induced ubiquitylation, but did not increase K625R ubiquitylation (Fig. 6f). Besides, K625 mutation blocked the degradation of MTP $\alpha$. Moreover, treatment with EX-527 could not further decrease the protein levels of K625 mutation (Fig. 6g). Our results suggest that acetylation of the K625 lysine site promoted MTP $\alpha$ ubiquitylation and degradation. 


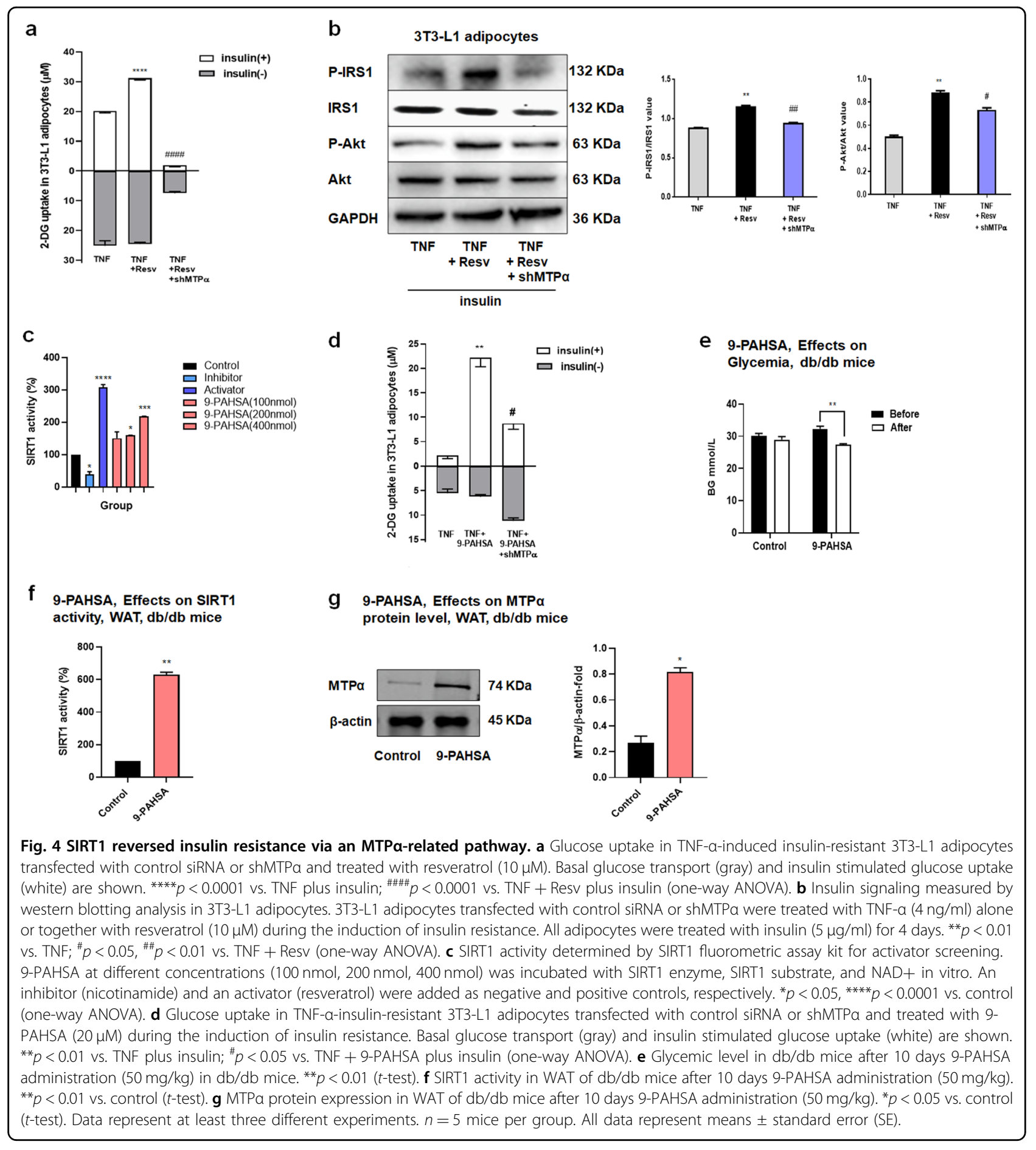

We found that K625 mutation, much like ovMTP $\alpha$, increased insulin-stimulated glucose uptake in TNF- $\alpha$ induced insulin-resistant cells, indicating that K625R improved IR. Moreover, EX-527 did not attenuate the effect of K625R mutation (Fig. 6h), suggesting that SIRT1 decreased IR by inhibiting MTP $\alpha$ ubiquitylation.

\section{Discussion}

MTP $\alpha$ is an important enzyme involved in FAO. Defective FAO produces excess free fatty acids, contributing to the development of IR. In this study, we identified a new function for MTP $\alpha$ in glucose metabolism. We found that MTP $\alpha$ expression was significantly 


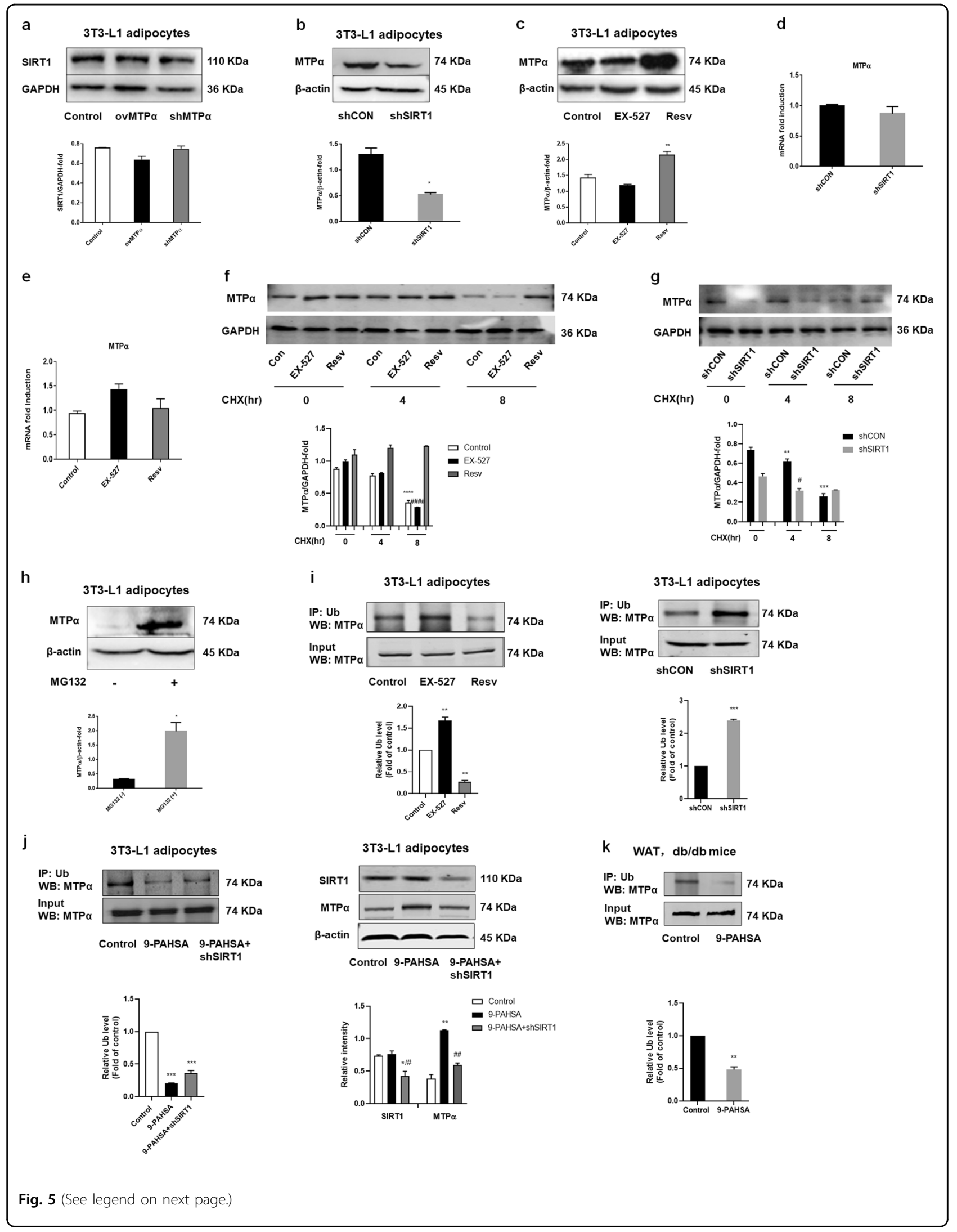


(see figure on previous page)

Fig. 5 SIRT1 increased MTPa protein levels by inhibiting MTPa ubiquitylation. a SIRT1 protein levels in 3T3-L1 adipocytes transfected with control siRNA, ovMTPa, or shMTPa. b MTPa protein levels in 3T3-L1 adipocytes transfected with control siRNA or shSIRT1. ${ }^{*} p<0.05$ vs. shCON ( $t$-test). c MTPa protein levels in 3T3-L1 adipocytes treated with EX-527 $(10 \mu \mathrm{M})$ or resveratrol $(10 \mu \mathrm{M})$ for 4 days. ${ }^{* *} p<0.01$ vs. control (one-way ANOVA). d MTPa mRNA levels in 3T3-L1 adipocytes transfected with control siRNA or shSIRT1. e MTPa mRNA levels in 3T3-L1 adipocytes treated with EX-527 $(10 \mu \mathrm{M})$ or resveratrol $(10 \mu \mathrm{M})$ for 4 days. f 3T3-L1 adipocytes treated with EX-527 $(10 \mu \mathrm{M})$ or resveratrol $(10 \mu \mathrm{M})$ for 4 days, then treated with $\mathrm{CHX}$ for

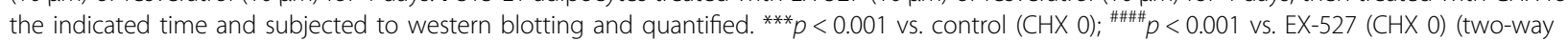
ANOVA). $\mathbf{g}$ 3T3-L1 adipocytes transfected with control siRNA or shSIRT1, treated with CHX for the indicated time and subjected to western blotting and quantified. ${ }^{* *} p<0.01,{ }^{* * *} p<0.001$ vs. control (CHX 0); ${ }^{*} p<0.001$ vs. shSIRT1 (CHX 0) (two-way ANOVA). h MTPa protein levels in 3T3-L1 adipocytes treated with $(+)$ or without $(-)$ MG132 $(10 \mu \mathrm{M})$ for $4 \mathrm{~h}$. ${ }^{*} p<0.05 \mathrm{vs}$. MG132 (-) (t-test). i 3T3-L1 adipocytes treated with EX-527 (10 $\left.\mu \mathrm{M}\right)$ or resveratrol $(10 \mu \mathrm{M})$ for 4 days, or transfected with control siRNA or shSIRT1. Then MTPa was immunoprecipitated with anti-Ub antibody from 3T3-L1 adipocytes and ubiquitylation levels assessed by western blotting. ${ }^{* *} p<0.01$ vs. control (one-way ANOVA). ${ }^{* *} p<0.001$ vs. shCON (t-test). $\mathbf{j}$ MTPa ubiquitylation and protein expression of SIRT1 and MTPa were measured in 3T3-L1 adipocytes transfected with control siRNA or shSIRT1 and treated with 9-PAHSA $(20 \mu \mathrm{M})$ for 4 days. ${ }^{*} p<0.05,{ }^{* *} p<0.01,{ }^{* * *} p<0.001$ vs. control, ${ }^{\#} p<0.05,{ }^{\# \#} p<0.01$ vs. 9-PAHSA (one-way ANOVA). k MTPa ubiquitylation measured in WAT of $\mathrm{db} / \mathrm{db}$ mice after 10 days 9 -PAHSA administration $(50 \mathrm{mg} / \mathrm{kg}) .{ }^{* *} p<0.01 \mathrm{vs}$. control (t test). Data represent at least three different experiments. $n=5$ mice per group. All data represent means \pm standard error (SE).

decreased in insulin-resistant cell models and diabetic mice. Importantly, MTP $\alpha$ overexpression reduced IR. In contrast, MTP $\alpha$ down-regulation promoted IR. We found that SIRT1 mediated this activity by deacetylating MTP $\alpha$ and inhibiting MTP $\alpha$ degradation.

Previous studies show that lysine acetylation is an important mechanism for regulating glucose homeostasis $^{21,22}$. Our findings support this previous work with the discovery that MTP $\alpha$ acetylation is a regulatory mechanism underlying IR. We identified four lysine sites (K383, K620, K359, and K625) on MTPa in insulinresistant 3T3-L1 adipocytes. SIRT1 reportedly protects against diet-induced insulin resistance ${ }^{23}$ and enhances insulin signaling in multiple types of insulin sensitive cells $^{24}$. In our study, SIRT1 deacetylated MTP $\alpha$ at the K625 site. Moreover, SIRT1 regulation of MTP $\alpha$ increased glucose uptake by deacetylating K625 on MTP $\alpha$. From these findings, we conclude that SIRT1-regulated MTP $\alpha$ deacetylation is crucial to maintain glucose homeostasis. Deacetylation and acetylation are known to affect enzyme activity. For example, deacetylation of trifunctional enzyme subunit alpha (ECHA) promotes $\mathrm{FAO}^{25}$. Lactate dehydrogenase $\mathrm{A}$ is acetylated at $\mathrm{K} 5$ and this acetylation inhibits its enzymatic activity ${ }^{26}$. SIRT5-mediated deacetylation of carbamoyl phosphate synthetase 1 increases its amino acid catabolism activity ${ }^{27}$. Hyperglycemia contributes to hyperacetylation of fatty acid $\beta$-oxidation enzymes, resulting in dysregulated energy metabolism ${ }^{28}$. Similar to these previous reports, we found that deacetylation of MTP $\alpha$ reduced IR. Our study showed that IR downregulated MTP $\alpha$. SIRT1 activation also affected MTP $\alpha$ protein levels, but not MTP $\alpha$ mRNA expression. Specifically, EX-527 treatment decreased MTP $\alpha$ protein expression, whereas resveratrol increased MTP $\alpha$ protein levels. This leads us to hypothesize that SIRT1 regulates MTP $\alpha$ degradation by regulating MTP $\alpha$ acetylation.

A previous study reported that a ubiquitin proteasome pathway degrades $\mathrm{MTP}^{14}$. The ubiquitin-proteasome system (UPS) is important for controlling levels of various cellular proteins and regulating degradation of mitochondrial proteins. We consistently found that treating 3T3-L1 adipocytes with the proteasomal inhibitor MG132 increased MTP $\alpha$ protein levels, suggesting that MTP $\alpha$ is a target of UPS. Studies report ${ }^{14}$ that MTP $\alpha$ acetylation prevents its ubiquitylation, suggesting that acetylation and ubiquitylation in MTP $\alpha$ may compete with each other by targeting the same lysine residues. However, we found that acetylation of MTP $\alpha$ promoted its ubiquitylation. The difference between our study and previous results may be due to different cell and insulin-resistant models. In our study, EX-527 increased MTPa ubiquitylation by inhibiting MTP $\alpha$ deacetylation in insulin-resistant 3T3-L1 adipocytes. K625 site mutation disrupted MTP $\alpha$ acetylation and ubiquitylation, and EX-527 treatment did not restore these modifications. Thus, our results support the idea that MTP $\alpha$ acetylation promoted its ubiquitylation. Besides, the lysine acetylation sites on MTP between the two studies are different. Liang Guo, et al. ${ }^{14}$ show that MTP $\alpha$ is acetylated at lysine residues 350/383/406 $(\mathrm{MTP} \alpha-3 \mathrm{~K})$, which promotes its protein stability by antagonizing its ubiquitylation on the three same lysines (MTP $\alpha-3 \mathrm{~K})$ and blocking its subsequent degradation. SIRT4 deacetylated MTP $\alpha$ at lysine residues 350/383/406 and destabilized it. In our study, we identified four lysineacetylated sites (K383, K620, K359, and K625) on MTPa by MS. SIRT1 deacetylated MTP $\alpha$ at the K625 site and repressed its degradation. Consistent with our findings, acetylation reportedly decreased ECHA protein stability, due to SIRT3 overexpression preventing ECHA degradation by decreasing its acetylation in $\beta$-cells ${ }^{25}$.

SIRT1 is a metabolic sensor with many roles in regulating cell biology, such as energy metabolism. However, the mechanism behind SIRT1 activity is not fully understood although it is known that SIRT1 NAD-dependent deacetylase activity mediates many of its functions. In our study, we found that SIRT1 also localized in mitochondria 


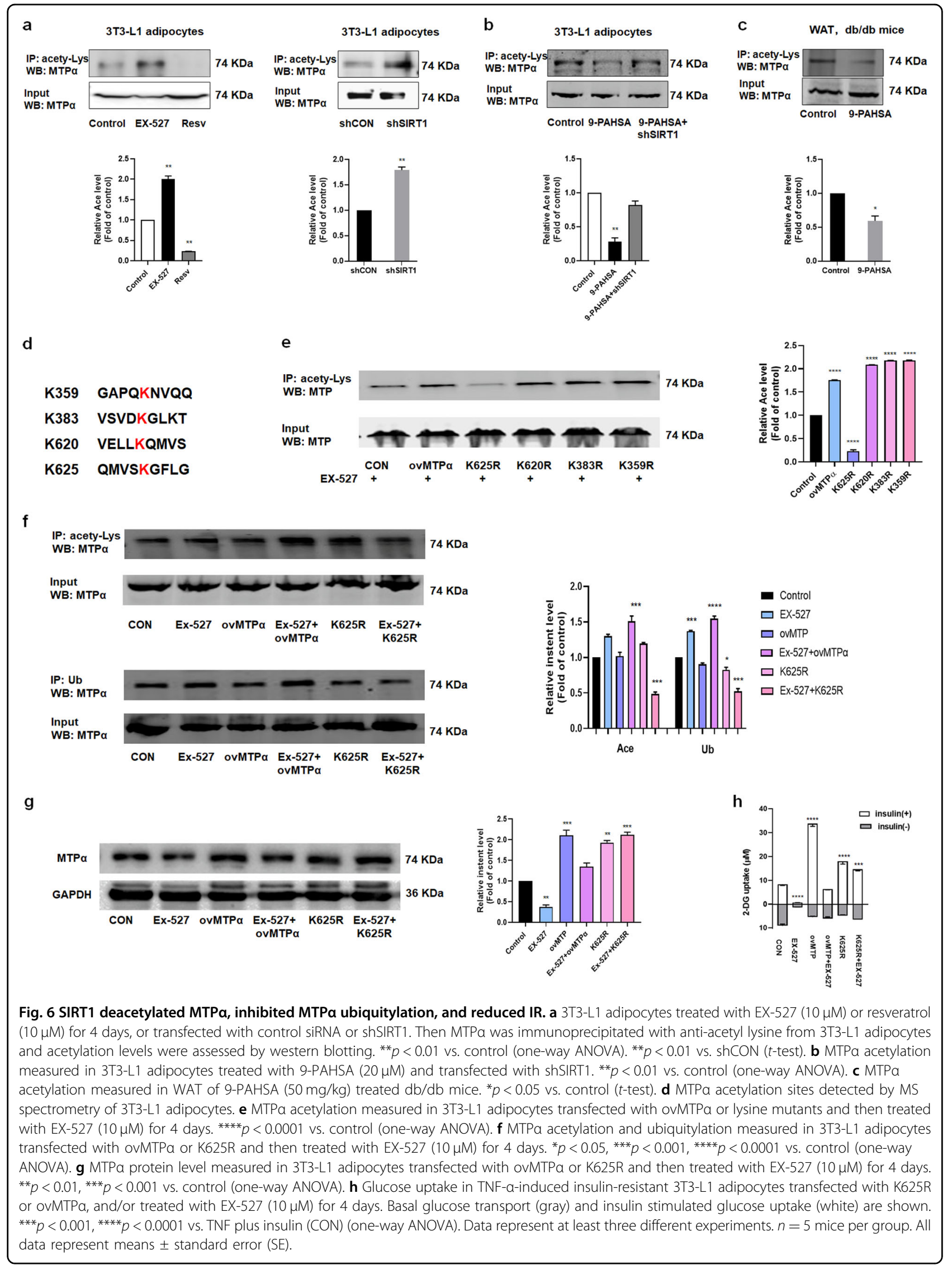




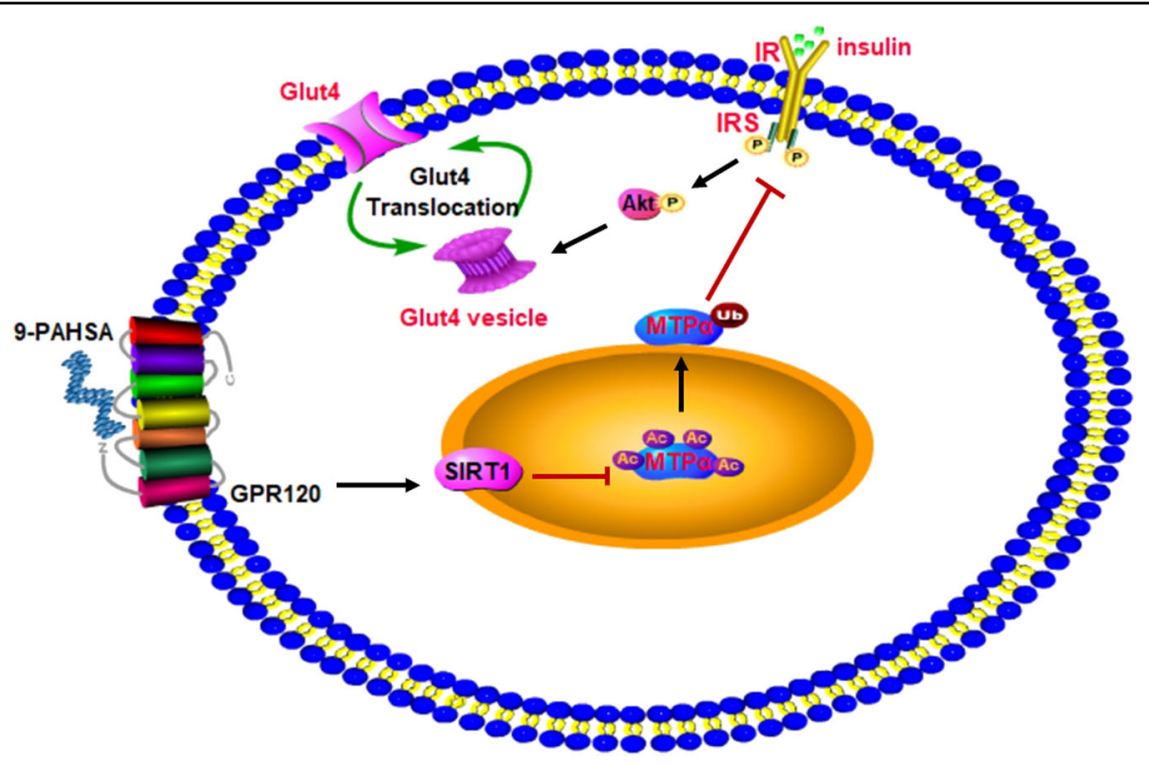

Fig. 7 Deacetylation of MTPa mediated by SIRT1 inhibited MTPa ubiquitylation and reduced insulin resistance. SIRT1 activation by 9-PAHSA decreased acetylation and ubiquitylation of MTPa, which inhibited MTPa degradation and activated the insulin signaling pathway.

in addition to the cytoplasm and the nucleus. SIRT1mediated deacetylation of MTP $\alpha$ at K625 prevented MTP $\alpha$ ubiquitylation. Moreover, SIRT1 knockdown in 3T3-L1 adipocytes downregulated MTP $\alpha$ protein levels. This finding was consistent with previous studies that showed that decreased SIRT1 expression caused lower MTP $\alpha$ protein expression ${ }^{29}$. We also found that K625 mutation enhanced insulin-dependent glucose uptake and reduced IR caused by TNF- $\alpha$ treatment. Together, these findings indicate that SIRT1 reduced IR by deacetylating MTP $\alpha$ and inhibiting MTP $\alpha$ degradation.

Resveratrol, a natural phenol found in the skin of grapes and blueberries, is a SIRT1 activator. Reportedly, resveratrol improves glucose control and insulin sensitivity in animal and cell culture studies ${ }^{19}$. However, the efficacy and safety of resveratrol treatment in humans requires further study. An alternative to resveratrol, 9-PAHSA, may activate SIRT1 and reduce MTP $\alpha$ acetylation. Notably, 9PAHSA is an endogenous fatty acid that reduces blood glucose levels and inflammation ${ }^{20}$. Thus, 9-PAHSA is a promising candidate for further therapeutic investigation.

In summary, we present a novel function for MTPa in reducing IR. The modification of MTP $\alpha$ by acetylation and ubiquitylation was crucial for regulating MTP $\alpha$ function in glucose metabolism (Fig. 7). SIRT1, a mitochondrial deacetylase, reduced MTP $\alpha$ acetylation and prevented MTP $\alpha$ degradation, resulting in increased insulin dependent glucose uptake in insulin-resistant cells. Therefore, 9PAHSA, a SIRT1 activator, is a new and promising means to reduce IR.

\section{Acknowledgements}

This work was supported by National Natural Science Foundation of China grant funded by the Chinese government (no. 81370360) and Shanghai Shen Kang Hospital Development Center (SHDC12015317).

\section{Competing interests}

The authors declare no competing interests.

\section{Publisher's note}

Springer Nature remains neutral with regard to jurisdictional claims in published maps and institutional affiliations.

Supplementary Information accompanies this paper at (https://doi.org/ 10.1038/s41419-020-03012-9).

Received: 7 April 2020 Revised: 8 September 2020 Accepted: 11 September 2020

Published online: 02 October 2020

\section{References}

1. Shanik, M. H. et al. Insulin resistance and hyperinsulinemia: is hyperinsulinemia the cart or the horse? Diabetes Care 31 Suppl 2, S262-S268 (2008).

2. Houstis, N., Rosen, E. D. \& Lander, E. S. Reactive oxygen species have a causal role in multiple forms of insulin resistance. Nature 440, 944-948 (2006).

3. Kusunoki, J., Kanatani, A. \& Moller, D. E. Modulation of fatty acid metabolism as a potential approach to the treatment of obesity and the metabolic syndrome. Endocrine 29, 91-100 (2006).

4. Zhang, Y. J. et al. Resveratrol ameliorates high-fat diet-induced insulin resistance and fatty acid oxidation via ATM-AMPK axis in skeletal muscle. Eur. Rev. Med. Pharm. Sci. 23, 9117-9125 (2019).

5. Guo, Q. et al. The saturated fatty acid palmitate induces insulin resistance through Smad3-mediated down-regulation of FNDC5 in myotubes. Biochem. Biophys. Res. Commun. 520, 619-626 (2019).

6. Rector, R. S. et al. Selective hepatic insulin resistance in a murine model heterozygous for a mitochondrial trifunctional protein defect. Hepatology 57, 2213-2223 (2013) 
7. Ibdah, J. A. et al. Mice heterozygous for a defect in mitochondrial trifunctional protein develop hepatic steatosis and insulin resistance. Gastroenterology $\mathbf{1 2 8}$ 1381-1390 (2005)

8. Sweet, I. R. et al. Endothelial inflammation induced by excess glucose is associated with cytosolic glucose 6-phosphate but not increased mitochondrial respiration. Diabetologia 52, 921-931 (2009).

9. Koziel, A., Woyda-Ploszczyca, A., Kicinska, A. \& Jarmuszkiewicz, W. The influence of high glucose on the aerobic metabolism of endothelial EA.hy926 cells. Pflug. Arch. 464, 657-669 (2012).

10. Scott, I., Webster, B. R., Li, J. H. \& Sack, M. N. Identification of a molecular component of the mitochondrial acetyltransferase programme: a novel role for GCN5L1. Biochem. J. 443, 655-661 (2012).

11. Baeza, J., Smallegan, M. J. \& Denu, J. M. Site-specific reactivity of nonenzymatic lysine acetylation. ACS Chem. Biol. 10, 122-128 (2015).

12. Guan, K. L. \& Xiong, Y. Regulation of intermediary metabolism by protein acetylation. Trends Biochem. Sci. 36, 108-116 (2011).

13. Choudhary, C., Weinert, B. T., Nishida, Y., Verdin, E. \& Mann, M. The growing landscape of lysine acetylation links metabolism and cell signalling. Nat. Rev. Mol. Cell Biol. 15, 536-550 (2014).

14. Guo, L. et al. Acetylation of mitochondrial trifunctional protein alpha-subunit enhances its stability to promote fatty acid oxidation and is decreased in nonalcoholic fatty liver disease. Mol. Cell Biol. 36, 2553-2567 (2016).

15. Liang, F., Kume, S. \& Koya, D. SIRT1 and insulin resistance. Nat. Rev. Endocrinol. 5, 367-373 (2009)

16. Li, F. et al. Adipose-specific knockdown of Sirt1 results in obesity and insulin resistance by promoting exosomes release. Cell Cycle 18, 2067-2082 (2019).

17. Liu, H. X., Wang, Y. M., Hu, J. P., Huang, L. Y. \& Fang, N. Y. Adipocyte differentiation is regulated by mitochondrial trifunctional protein alpha-subunit via sirtuin 1. Exp. Cell Res. 357, 271-281 (2017).

18. Wang, Y. M., Liu, H. X. \& Fang, N. Y. High glucose concentration impairs 5PAHSA activity by inhibiting AMP-activated protein kinase activation and promoting nuclear factor-kappa-B-mediated inflammation. Front. Pharm. 9 1491 (2018).

19. Cote, C. D. et al. Resveratrol activates duodenal Sirt1 to reverse insulin resistance in rats through a neuronal network. Nat. Med. 21, 498-505 (2015).

20. Yore, M. M. et al. Discovery of a class of endogenous mammalian lipids with anti-diabetic and anti-inflammatory effects. Cell 159, 318-332 (2014).

21. Yoshino, J., Mills, K. F., Yoon, M. J. \& Imai, S. Nicotinamide mononucleotide, a key $\mathrm{NAD}(+)$ intermediate, treats the pathophysiology of diet- and ageinduced diabetes in mice. Cell Metab. 14, 528-536 (2011).

22. Canto, $C$. et al. The $\mathrm{NAD}(+)$ precursor nicotinamide riboside enhances oxidative metabolism and protects against high-fat diet-induced obesity. Cell Metab. 15, 838-847 (2012).

23. Sun, C. et al. SIRT1 improves insulin sensitivity under insulin-resistant conditions by repressing PTP1B. Cell Metab. 6, 307-319 (2007).

24. Shu, Q. et al. Acupuncture targeting SIRT1 in the hypothalamic arcuate nucleus can improve obesity in high-fat-diet-induced rats with insulin resistance via an anorectic effect. Obes. Facts 13, 40-57 (2020).

25. Zhang, Y. et al. The pivotal role of protein acetylation in linking glucose and fatty acid metabolism to beta-cell function. Cell Death Dis. 10, 66 (2019).

26. Zhao, D. et al. Lysine-5 acetylation negatively regulates lactate dehydrogenase A and is decreased in pancreatic cancer. Cancer Cell 23, 464-476 (2013).

27. Nakagawa, T., Lomb, D. J., Haigis, M. C. \& Guarente, L. SIRT5 deacetylates carbamoyl phosphate synthetase 1 and regulates the urea cycle. Cell 137 560-570 (2009).

28. Kumar, S. et al. Sirtuin1-regulated lysine acetylation of p66Shc governs diabetes-induced vascular oxidative stress and endothelial dysfunction. Proc. Natl Acad. Sci. USA 114, 1714-1719 (2017).

29. Garcia, M. M. et al. Methyl donor deficiency induces cardiomyopathy through altered methylation/acetylation of PGC-1a by PRMT1 and SIRT1. J. Pathol. 225 324-335 (2011). 